\title{
Traitement du Trouble Bipolaire en Phase Maniaque : synthèse critique des recommandations internationales.
}

\section{Bipolar Disorder Treatment of manic phases : critical synthesis from international guidelines.}

Pierre Alexis GEOFFROY ${ }^{1,5,6,7^{*}}$, Frank BELLIVIER ${ }^{1,2,4,7}$, Chantal HENRY ${ }^{1,3,5,7}$.

1) INSERM, U955, Psychiatrie génétique, Créteil, 94000, France ;

2) Université Paris Diderot, Faculté de médecine, Paris, 75013, France ;

3) Université Paris Est, Faculté de médecine, Créteil, 94000, France ;

4) AP-HP, Hôpital St Louis-Lariboisière-F. Widal, Pôle de Psychiatrie, Paris, 75475, France ;

5) AP-HP, Hôpital H. Mondor - A. Chenevier, Pôle de Psychiatrie, Créteil, 94000, France ;

6) Pôle de psychiatrie, Université Lille Nord de France, CHRU de Lille, F-59000 Lille, France;

7) Fondation FondaMental, Créteil, 94000, France.

\section{*Correspondance à l'auteur :}

Pierre Alexis GEOFFROY,

INSERM - U955 équipe 15 Psychiatrie Génétique

Pôle de Psychiatrie, Centre Expert Bipolaire, pavillon hartman

Hôpital Albert Chenevier, 40 rue de Mesly

94000 Créteil Cedex - FRANCE

Tel: $+\underline{33149813290}$ - Fax: $+\underline{33149813099}$

pierre.a.geoffroy@gmail.com

Compte total : 15 pages /15

(résumés, texte, tableaux et bibliographie).

Conflits d'intérêt : Les auteurs n'ont aucun conflit d'intérêt pouvant influencer une partie ou la totalité de ce travail.

Keywords: bipolar disorder, guidelines, treatment, mood stabilizer, mania, manic episode.

Mots-clefs : trouble bipolaire, recommandations, traitement, thymorégulateur, manie, épisode maniaque. 


\section{Résumé francais :}

Le Trouble Bipolaire est une maladie récurrente sévère reconnue par l'Organisation Mondiale de la Santé (OMS) comme la sixième cause de handicap par année de vie parmi toutes les maladies dans la population des 15 à 44 ans. Le handicap majeur conféré par cette pathologie est lié au très haut niveau de rechute, à l'impact fonctionnel des comorbidités associées et des troubles cognitifs entre les épisodes. L'utilisation systématique par les cliniciens d'algorithmes décisionnels issus des recommandations améliore la prise en charge globale des patients bipolaires, en comparaison à une modalité de traitement habituel. Bien qu'elles n'embrassent pas l'ensemble des situations rencontrées avec les patients, cette approche systématique contribue au développement d'une médecine personnalisée, indispensable dans cette pathologie. Notre revue synthétise les recommandations internationales les plus récentes dans la prise en charge des phases maniaques. De manière consensuelle, les recommandations proposent en première intention l'utilisation des stabilisateurs de l'humeur traditionnels (lithium ou valproate) et des antipsychotiques atypiques (APA - olanzapine, rispéridone, aripiprazole et quétiapine), ainsi que l'arrêt des antidépresseurs. Les recommandations sont divergentes concernant l'utilisation des combinaisons thérapeutiques proposées soit en traitement de première intention pour les cas sévères, soit en seconde intention. Le traitement des états mixtes est aussi peu consensuel et quelques recommandations proposent le valproate, la carbamazépine et certains APA en première intention, en déconseillant l'utilisation du lithium. Enfin, la durée du traitement des phases maniaques est non consensuelle.

\section{Résumé anglais:}

Introduction: Bipolar Disorder (BD) is the sixth leading cause of disability per year of life among all diseases in the population 15 to 44 years. It is a group of heterogeneous diseases, with frequent comorbid psychiatric or somatic disorders, variable treatment response and frequent residual symptoms between episodes. The major impairment associated by this disorder is related to high relapse rates, the functional impact of comorbidities and cognitive impairment between episodes. The prognosis of the disease relies on the efficacy of relapse prevention interventions. Given the heterogeneity of the disorder, relapse prevention needs to develop a personalized care plan as soon as the acute phase. In such a complex situation, guidelines driven algorithms of decision are known to improve overall care of patients with bipolar disorder, compared to standard treatment decisions. Although guidelines do not account for all the situations encountered with patients, this systematic approach contributes to the development of personalized medicine. 
Methods: We present a critical review of recent international recommendations for the management of manic phases. We summarize treatment options that reach consensus (monotherapy and combination therapy) and comment on options that differ across guidelines.

Results: The synthesis of recent international guidelines shows a consensus for the initial treatment for manic phases. For acute and long-term management, the anti-manic drugs proposed are traditional mood stabilizers (lithium or valproate) and atypical antipsychotics (APA - olanzapine, risperidone, aripiprazole and quetiapine). All guidelines indicate to stop antidepressant drugs during manic phases. International guidelines also present with some differences. First, as monotherapy is often non sufficient in clinical practice, combination therapy with a traditional mood stabilizer and an APA are disputed either in first line treatment for severe cases or in second line. Second, mixt episodes treatment is not consensual too and some guidelines propose in first line valproate, carbamazepine and some APA and advice not to use lithium. On the other hand, some guidelines do not propose specific treatment for mixt episodes and group it with manic episodes management. Duration of treatment is unclear.

Conclusion: Guidelines utilization has shown that the systemic use by clinicians of decision algorithms in comparison to "treatment as usual" modality improves the overall care of patients with BD. Future data from cohort of patients seem necessary to complement the existing data from clinical trials. These cohort studies will help to take into account the different individual profiles of $\mathrm{BD}$ and thus may help to propose a more personalized medicine.

\section{Introduction}

Le Trouble Bipolaire (TB) est une maladie récurrente sévère reconnue par l'Organisation Mondiale de la Santé (OMS) comme la sixième cause de handicap par année de vie parmi toutes les maladies dans la population des 15 à 44 ans [1]. On observe une très grande hétérogénéité de la maladie, avec de fréquentes comorbidités psychiatriques ou somatique, de réponse aux traitements ou de symptômes résiduels entre les épisodes [2]. Le handicap majeur conféré par cette pathologie est lié au très haut niveau de rechute, à l'impact fonctionnel des comorbidités associées et des troubles cognitifs entre les épisodes. Le pronostic de la pathologie est donc à l'efficacité des mesures prophylactiques et à la prise en compte de cette 
hétérogénéité pour la définition d'un programme de soins personnalisés qui doit être élaboré dès la phase aigue [3]. Les recommandations thérapeutiques actuelles proposent l'instauration d'un traitement thymorégulateur (ou stabilisateurs de l'humeur) dès le premier épisode maniaque car $90 \%$ évoluent vers un TB [4]. Les indications des thymorégulateurs dans le TB sont issues des recommandations professionnelles, ou «guidelines », qui sont des propositions de prise en charge thérapeutiques développées à partir d'une évaluation méthodique de la littérature scientifique internationale conjuguée à des avis d'experts. Elles permettent d'orienter les cliniciens dans leur choix thérapeutique en facilitant l'accès aux données de la littérature scientifique et en les synthétisant selon une médecine dite «basée sur les preuves ». Cependant ces recommandations ne suivent pas toutes la même méthodologie et même si certains points sont consensuels, d'autres le sont moins et ne facilitent pas leur compréhension et leur applicabilité clinique.

Ainsi, notre revue se propose de synthétiser les principales recommandations internationales les plus récentes dans la prise en charge des phases maniaques. Ceci est d'autant plus pertinent qu'il n'existe pas à ce jour de recommandations françaises suivant ces méthodes. Nous présenterons en premier lieu les options thérapeutiques (monothérapie et bithérapie) faisant consensus, puis seront discutés les points de divergence pour enfin réaliser un résumé critique.

\section{Méthodes}

Cette synthèse s'appuiera sur les recommandations internationales de la version collaborative récemment mise à jour du réseau canadien (CANMAT, Canadian Network for Mood and Anxiety) et de la société international des troubles bipolaires (ISBD, International Society for Bipolar Disorders) publiée en 2009 [5], de celles de la fédération mondiale des sociétés de psychiatrie biologique (WFSBP, World Federation of Societies of Biological Psychiatry 2009 [6]), de celles l'association britannique de psychopharmacologie (BAP, British Association for Psychopharmacology [7] de 2009), les recommandations très pragmatiques de la National Institute for Health and Clinical Excellence (NICE) de 2006 [4], et enfin les recommandations australiennes [8]. Certaines recommandations plus anciennes seront citées afin de comprendre les changements survenus au cours des dernières années [9$12]$.

En France, seules des recommandations professionnelles fondées sur les pratiques basées sur l'expérience d'un panel d'experts, sont disponibles. Cette méthodologie est complémentaire 
des recommandations fondées sur les preuves et apportent des indications pour des situations cliniques pour lesquelles les données d'études contrôlées randomisées sont rares [13].

Par ailleurs, soulignons qu'il existe une différence théorique des notions de rechute (ou « relapse » en anglais) et récidive (ou « recurrence » en anglais) renvoyant respectivement à un traitement d'entretien (instauré pour éviter une rechute ou résurgence des symptômes d'un épisode thymique non terminé) et à un traitement préventif des récidives (traitement à distance de l'épisode thymique et instauré pour éviter un nouvel épisode). Dans la plupart des recommandations thérapeutiques cette distinction n'est pas effectuée et apparaît en l'état actuel de la science purement théorique avec en pratique un recouvrement important des deux notions [14]. Aussi, nous n'avons pu et ne faisons pas la distinction de ces deux notions dans cette revue de littérature.

Nous avons ainsi effectué une synthèse critique des recommandations internationales pour y dégager les consensus et d'en discuter les divergences afin d'aider le praticien dans ses choix thérapeutiques. Cette synthèse se centrera sur les molécules disponibles en France et ayant reçu une autorisation de mise sur le marché dans l'indication du traitement des troubles bipolaires (HAS) [15].

\section{3- Indications des thymorégulateurs dans les phases maniaques}

La prescription d'un thymorégulateur dans le trouble bipolaire doit s'accompagner d'une information sur les traitements et d'une prise en charge centrée sur des conseils d'hygiène de vie et des mesures psycho-éducatives [4]. L'information relative aux effets secondaires et aux mesures de surveillance est également primordiale. Ces mesures réduisent le risque élevé de rupture de traitement et renforce l'alliance thérapeutique en faveur d'une meilleure observance.

\subsection{Options thérapeutiques consensuelles}

\subsubsection{Traitement en phase aigue}

De manière consensuelle, toutes les recommandations s'accordent pour interrompre un traitement antidépresseur en première intention. Il est également recommandé d'associer à la prescription d'un thymorégulateur de mesures telles que l'éviction des stimulants environnementaux, la préconisation d'activités calmes et la ritualisation du quotidien comprenant peu d'activités [4]. 
Il existe un relatif consensus pour préconiser une monothérapie en première intention [4-8]. Ces recommandations proposent les mêmes molécules en première ligne dans la prise en charge d'un épisode maniaque [4-8]: le lithium (Li), l'acide valproïque (VPA) et les antipsychotiques atypiques (APA ou antipsychotiques dits de seconde génération) olanzapine, rispéridone, aripiprazole et quétiapine. Le tableau 1 résume les recommandations issues du CANMAT-ISBD pour les molécules commercialisées en France ou en cours de demande d'AMM.

Tableau 1 : Recommandations pour le traitement des épisodes maniaques (CANMAT et ISBD, 2009). Adaptées pour les molécules commercialisées en France ou en cours de demande d'AMM.

\begin{tabular}{|c|l|}
\hline Option 1 & $\begin{array}{l}\text {-lithium } \\
\text {-valproate } \\
\text {-antipsychotique atypique (olanzapine, risperidone, quetiapine, } \\
\text { aripiprazole) } \\
\text {-lithium ou valproate + olanzapine/risperidone/quetiapine/aripiprazole }\end{array}$ \\
\hline Option 2 & $\begin{array}{l}\text {-carbamazépine } \\
\text {-lithium + valproate } \\
\text {-asenapine } \\
\text {-asenapine + lithium ou valproate } \\
\text {-électro-convulsivo-thérapie (ECT) }\end{array}$ \\
\hline Option 3 & $\begin{array}{l}\text {-halopéridol, chlorpromazine, } \\
\text {-lithium ou valproate + halopéridol } \\
\text {-lithium + carbamazépine } \\
\text {-clozapine }\end{array}$ \\
\hline Non & $\begin{array}{l}\text {-monothérapie avec gabapentine, topiramare, lamotrigine } \\
\text {-rispéridone + carbamazépine } \\
\text {-olanzapine + carbamazépine }\end{array}$ \\
\hline
\end{tabular}

\subsubsection{Efficacité et profil de tolérance : deux ingrédients de la réussite thérapeutique.}

Nous avons vu que le facteur principal de rechute et d'échec thérapeutique était l'inobservance et l'arrêt prématuré des thymorégulateurs [16]. Au delà des facteurs directement liés au patient, il y a ceux imputables aux effets secondaires des traitements dont il conviendra d'informer le patient. Finalement, c'est l'efficacité et le profil de tolérance qui permettront de guider les prescriptions. Les recommandations de la WFSBP proposent une synthèse tenant compte de ces deux aspects pour chaque molécule. Nous rapportons dans le tableau 2 un résumé utile pour la pratique clinique qui tient compte des posologies recommandées [6]. 
Tableau 2 : Niveau de preuve et recommandations tenant compte du profil de tolérance pour les traitements pharmacologiques et non pharmacologiques dans les manies et posologies recommandées. (Adapté de la WFSBP, 2009).

\begin{tabular}{|c|c|c|c|}
\hline Traitement & $\begin{array}{c}\text { Niveau de } \\
\text { certitude }\end{array}$ & $\begin{array}{c}\text { Niveau de } \\
\text { recommandation }\end{array}$ & $\begin{array}{c}\text { Posologie } \\
\text { recommandée }\end{array}$ \\
\hline Aripiprazole & A & $\mathbf{1}$ & $15-30 \mathrm{mg}$ \\
\hline Azenapine & A & 2 & $10-20 \mathrm{mg}$ \\
\hline Carbamazepine & A & 2 & $600-1200 \mathrm{mg}$ \\
\hline Haloperidol & A & 2 & $5-20 \mathrm{mg}$ \\
\hline Lithium & A & 2 & $600-1200 \mathrm{mg}$ \\
\hline Olanzapine & A & 2 & $10-20 \mathrm{mg}$ \\
\hline Quetiapine & A & 2 & $400-800 \mathrm{mg}$ \\
\hline Risperidone & A & $\mathbf{1}$ & $2-6 \mathrm{mg}$ \\
\hline Valproate & A & $\mathbf{1}$ & $1200-3000 \mathrm{mg}$ \\
\hline Chlorpromazine & B & 3 & $300-1000 \mathrm{mg}$ \\
\hline Amisulpride & C1 & 4 & $400-800 \mathrm{mg}$ \\
\hline Clonazepam & C1 & 4 & $2-8 \mathrm{mg}$ \\
\hline Clozapine & $\mathrm{C} 1$ & 4 & $100-300 \mathrm{mg}$ \\
\hline Lorazepam & $\mathrm{C} 1$ & 4 & 4 à $8 \mathrm{mg}$ \\
\hline Oxcarbazepine & $\mathrm{C} 1$ & 4 & $900 \mathrm{à} 1800 \mathrm{mg}$ \\
\hline Lamotrigine & $\mathrm{E}$ & - & $50 \mathrm{à} 200 \mathrm{mg}$ \\
\hline ECT & $\mathrm{C} 1$ & 4 & \\
\hline rTMS & $\mathrm{E}$ & - & \\
\hline
\end{tabular}

ECT = électro-convulsivo-thérapie, rTMS = stimulation magnétique transcranienne répétée

\subsection{Recommandations internationales : points de divergences et synthèse critique}

\subsubsection{Durée du traitement}

Si la durée du traitement est une question fréquemment posée par les patients, l'absence de niveau de preuve fait qu'il n'y a que de très rares recommandations sur le sujet.

Le NICE recommande de poursuivre un traitement prophylactique au moins 2 ans après un premier épisode thymique ou au moins 5 ans si le patient présente des risques de récidive importants tels que : un épisode psychotique sévère, une comorbidité addictive, un isolement social, une situation de précarité [4]. La WFSBP recommandent la poursuite aussi longtemps que possible dès lors que le diagnostic de trouble bipolaire est posé [6].

L'objectif du traitement au long cours est d'achever la guérison de l'épisode thymique (en évitant la rechute) et d'en prévenir la récidive [9]. Le problème majeur du traitement au long cours est l'adhésion du patient au traitement. En effet, la plupart des patients rechutent à cause d'une absence totale ou partielle d'observance à ce traitement [16]. Parmi les facteurs favorisant l'observance, il existe des facteurs directement liés au patient tel que le fait d'avoir un bon niveau d'éducation et des facteurs liés aux traitements qui seront d'autant mieux suivis 
lorsqu'ils réduisent la sévérité des épisodes dépressifs, qu'ils n'entrainent pas de prise de poids ni de troubles cognitifs [16].

$\mathrm{Au}$ delà de la mauvaise observance, il existe également d'autres facteurs prédictifs de rechutes. Les plus importants sont les symptômes résiduels tant dépressifs que maniaques [17]. Ainsi, il est nécessaire de réduire au maximum les symptômes résiduels ce qui constituera par la suite un élément essentiel du pronostic du patient traité. Par ailleurs, des antécédents de cycles rapides constituent également un risque plus important de rechutes [18].

Les principaux effets secondaires à cours et long terme des différentes molécules participeront au choix du traitement. Le lithium est associé à un risque accru de défaut de filtration rénale, d'hypothyroïdie, d'hyperparathyroïdie et de prise de poids. Il existe cependant peu de preuves pour son imputabilité dans la survenue d'insuffisance rénale. Le risque de malformations congénitales est également revu à la baisse et le risque bénéfice/risque doit être évalué avec attention avant de prendre la décision de retirer le lithium au cours de la grossesse [19].

À l'inverse, l'acide valproïque reste en principe à éviter chez les femmes en âge de procréer à cause de son fort potentiel tératogène dose dépendant [20] et du risque de survenue d'ovaires polykystiques [4]. D’autre part la carbamazépine n'est plus un traitement de première intention à cause de ses effets secondaires.

L'utilisation des antipsychotiques au long cours est à évaluer en fonction du risque de survenue d'effets secondaires tels que l'apparition d'un diabète, d'un syndrome métabolique ou le risque plus élevé qu'avec les autres molécules d'évolution vers un épisode dépressif et ce particulièrement chez le sujet âgé. L'utilisation d'un antipsychotique peut également au long cours être pourvoyeur de symptomatologie négative secondaire avec notamment une abrasion des affects, entrainant notamment une mauvaise récupération fonctionnelle mais ceci est plus marqué avec les neuroleptiques classiques [21].

\subsubsection{Les associations thérapeutiques}

Plus récemment, des données ont clairement établit la supériorité des combinaisons de traitement avec le Li ou le VPA et un APA dans le traitement des phases maniaques [22]. À l'heure actuelle, ces associations sont réservées dans les recommandations internationales aux manies sévères (dans les recommandations du World Federation of Societies of Biological Psychiatry WFSBP [6] et la British Association for Psychopharmacology BAP [7]) et, comme seconde option thérapeutique dans les manies légères à modérées, y compris dans les 
recommandations de la haute autorité de santé HAS [15,23]. Cependant, le Canadian Network for Mood and Anxiety Treatments and International Society for Bipolar Disorders (CANMAT ISBD) préconisent la bithérapie Li ou VPA et un APA parmi les stratégies de première ligne sans préciser cette notion de sévérité de l'épisode [5].

Or en pratique clinique les patients bénéficient en grande majorité de plusieurs agents antimaniaques [22]. Ainsi, l'utilisation des associations thérapeutiques dans le cadre des phases maniaques est une pratique courante et largement prépondérante sur la monothérapie. De plus, il existe des discordances importantes entre les recommandations et les habitudes de prescription dans la maladie bipolaire puisque seulement un patient sur six est traité en fonction des recommandations [24]. En pratique, la monothérapie n'est souvent pas suffisante pour obtenir une réduction rapide des symptômes. Ainsi, moins de $10 \%$ des patients reçoivent une monothérapie pour le traitement des épisodes maniaques [23] et Wolsfsperger et al. rapportent une moyenne de plus de trois psychotropes prescrits au cours des états maniaques [25].

Ce décalage entre les recommandations internationales et la pratique clinique est probablement dû à la complexité et à la sévérité de cette maladie, à ses comorbidités, à l'inobservance thérapeutique et aux effets indésirables [26]. De plus, pour des raisons évidentes d'éthiques, les patients inclus dans les essais thérapeutiques contre placebo ne sont pas toujours représentatifs des patients hospitalisés dans nos services.

Une revue récente observe que de manière générale les antipsychotiques sont très largement prescrits : $72 \%$ à $92 \%$ des situations d'épisode maniaque [27]. La méta-analyse de Smith et al., portant sur 3089 sujets présentant un épisode maniaque, montre que les combinaisons avec haloperidol, olanzapine, risperidone et quetiapine par rapport aux monothérapies avec thymorégulateurs classiques sont significativement plus efficaces bien que moins bien tolérées [28]. Une revue récente réalisée par notre équipe sur les combinaisons thérapeutiques dans les phases maniaques confirme également, à la lecture de la littérature internationale, une efficacité supérieure des combinaisons Li ou VPA avec un APA [22].

Il faut souligner que malgré un profil de tolérance acceptable des bithérapies, ces profils sont variables en fonction des associations (voir revue de Geoffroy et collaborateurs [22] pour plus de détails). Néanmoins les combinaisons thérapeutiques sont généralement marquées par un taux d'effets secondaires et d'arrêts plus importants [22]. Le tableau 3 résume les combinaisons testées dans la littérature internationale [29-52]. 
Tableau 3 : Résumé des études issues de la littérature internationale testant les combinaisons thérapeutiques dans les épisodes maniaques (adapté de Geoffroy et al, 2012 [22]).

\begin{tabular}{|c|c|c|}
\hline Études & $\begin{array}{c}\text { Combinaison } \\
\text { traitement } \\
\text { (Nombre de sujets) }\end{array}$ & Résultats \\
\hline Garfinkel et al., 1980 & $\begin{array}{l}\mathrm{Li}+\mathrm{PBO}(7) \\
\mathrm{HAL}+\mathrm{PBO}(7) \\
\mathrm{Li}+\mathrm{HAL}(7)\end{array}$ & $\begin{array}{c}\mathrm{HAL}+\mathrm{PBO}=\mathrm{HAL}+\mathrm{Li} \\
\mathrm{HAL}+\mathrm{PBO}>\mathrm{PBO}+\mathrm{Li} \\
\mathrm{HAL}+\mathrm{Li}>\mathrm{PBO}+\mathrm{Li}\end{array}$ \\
\hline Klein et al., 1984 & $\begin{array}{l}\text { CBZ + HAL (14) } \\
\text { PBO + HAL (13) }\end{array}$ & $\mathrm{CBZ}+\mathrm{HAL}>\mathrm{PBO}+\mathrm{HAL}$ \\
\hline Müller \& Stoll, 1984 & $\begin{array}{l}\text { CBZ + HAL (6) } \\
\text { PBO + HAL }\end{array}$ & $\mathrm{CBZ}+\mathrm{HAL}>\mathrm{PBO}+\mathrm{HAL}$ \\
\hline Desai et al., 1987 & $\begin{array}{l}\mathrm{CBZ}+\mathrm{Li}(5) \\
\mathrm{PBO}+\mathrm{Li}\end{array}$ & $\mathrm{CBZ}+\mathrm{Li}>\mathrm{PBO}+\mathrm{Li}$ \\
\hline Möller et al., 1989 & $\begin{array}{l}\mathrm{CBZ}+\mathrm{HAL}(11) \\
\mathrm{PBO}+\mathrm{HAL}(9)\end{array}$ & $\begin{array}{c}\mathrm{CBZ}+\mathrm{HAL}=\mathrm{PBO}+\mathrm{HAL} \\
(\mathrm{CBZ}+\mathrm{HAL} \text { a moins consommé } \mathrm{LEV}=\text { preuve indirecte combinaison > } \\
\text { monothérapie })\end{array}$ \\
\hline Anand et al., 1999 & $\begin{array}{l}\mathrm{LAM}+\mathrm{Li}(8) \\
\mathrm{PBO}+\mathrm{Li}(8)\end{array}$ & $\mathrm{LAM}+\mathrm{Li}=\mathrm{PBO}+\mathrm{Li}$ \\
\hline $\begin{array}{l}\text { Müller-Oerlinghausen et al. } \\
2000\end{array}$ & $\begin{array}{l}\mathrm{VPA}+\mathrm{APT}(69) \\
\mathrm{PBO}+\mathrm{APT}(67)\end{array}$ & $\begin{array}{c}\mathrm{VPA}+\mathrm{APT}>\mathrm{PBO}+\mathrm{APT} \\
\mathrm{VPA}+\mathrm{SND} \text { permirent moins de prescription de } \mathrm{BZD} \text { et/ou APT }\end{array}$ \\
\hline Pande et al., 2000 & $\begin{array}{l}\mathrm{GBP}+\mathrm{SH}(54) \\
\mathrm{PBO}+\mathrm{SH}(59)\end{array}$ & $\mathrm{GBP}+\mathrm{SH}<\mathrm{PBO}+\mathrm{SH}$ \\
\hline Sachs et al., 2002 & $\begin{array}{l}\mathrm{RSP}+\mathrm{SH}(52) \\
\mathrm{HAL}+\mathrm{SH}(53) \\
\mathrm{PBO}+\mathrm{SH}(51)\end{array}$ & $\begin{array}{l}\mathrm{RSP}+\mathrm{SH}>\mathrm{PBO}+\mathrm{SH} \\
\mathrm{HAL}+\mathrm{SH}>\mathrm{PBO}+\mathrm{SH} \\
\mathrm{RSP}+\mathrm{SH}=\mathrm{HAL}+\mathrm{SH}\end{array}$ \\
\hline Tohen et al., 2002 & $\begin{array}{l}\mathrm{OLZ}+\mathrm{SH}(220) \\
\mathrm{PBO}+\mathrm{SH}(114)\end{array}$ & $\mathrm{OLZ}+\mathrm{SH}>\mathrm{PBO}+\mathrm{SH}$ \\
\hline Delbello et al., 2002 & $\begin{array}{l}\text { QTP + VPA (15) } \\
\text { PBO + VPA (15) }\end{array}$ & $\mathrm{QTP}+\mathrm{VPA}>\mathrm{PBO}+\mathrm{VPA}$ \\
\hline Yatham et al., 2003 & $\begin{array}{l}\mathrm{RSP}+\mathrm{SH}(75) \\
\mathrm{PBO}+\mathrm{SH}(75)\end{array}$ & $\mathrm{RSP}+\mathrm{SH}>\mathrm{PBO}+\mathrm{SH}$ \\
\hline Sachs et al., 2004 & $\begin{array}{l}\mathrm{QTP}+\mathrm{SH}(91) \\
\mathrm{PBO}+\mathrm{SH}(100)\end{array}$ & $\mathrm{QTP}+\mathrm{SH}>\mathrm{PBO}+\mathrm{SH}$ \\
\hline Akhondzadeh et al., 2006 & $\begin{array}{l}\text { ALP + HAL + Li (38) } \\
\text { PBO + HAL + Li (37) }\end{array}$ & $\mathrm{ALP}+\mathrm{HAL}+\mathrm{Li}>\mathrm{PBO}+\mathrm{HAL}+\mathrm{Li}$ \\
\hline McIntyre et al., 2007 & $\begin{array}{l}\mathrm{QTP}+\mathrm{SH}(197) \\
\mathrm{PBO}+\mathrm{SH}(205)\end{array}$ & $\mathrm{QTP}+\mathrm{SH}>\mathrm{PBO}+\mathrm{SH}$ \\
\hline Yatham et al., 2007 & $\begin{array}{l}\mathrm{QTP}+\mathrm{SH}(104) \\
\mathrm{PBO}+\mathrm{SH}(96)\end{array}$ & $\mathrm{QTP}+\mathrm{SH}>\mathrm{PBO}+\mathrm{SH}$ \\
\hline Sussmam et al., 2007 & $\begin{array}{l}\mathrm{QTP}+\mathrm{SH}(197) \\
\mathrm{PBO}+\mathrm{SH}(205)\end{array}$ & $\mathrm{QTP}+\mathrm{SH}>\mathrm{PBO}+\mathrm{SH}$ \\
\hline Vieta et al., 2008 & $\begin{array}{l}\mathrm{ARI}+\mathrm{SH}(253) \\
\mathrm{PBO}+\mathrm{SH}(131)\end{array}$ & $\mathrm{ARI}+\mathrm{SH}>\mathrm{PBO}+\mathrm{SH}$ \\
\hline Tohen et al., 2008 & $\begin{array}{l}\text { OLZ + CBZ (58) } \\
\text { PBO + CBZ (60) }\end{array}$ & $\mathrm{OLZ}+\mathrm{CBZ}=\mathrm{PBO}+\mathrm{CBZ}$ \\
\hline Juruena et al., 2009 & $\begin{array}{l}\mathrm{OXC}+\mathrm{Li}(26) \\
\mathrm{CBZ}+\mathrm{Li}(26)\end{array}$ & $\mathrm{OXC}+\mathrm{Li}>\mathrm{CBZ}+\mathrm{Li}$ \\
\hline Amrollahi et al., 2011 & $\begin{array}{l}\text { TXF + Li (20) } \\
\mathrm{PBO}+\mathrm{Li}(20)\end{array}$ & $\mathrm{TXF}+\mathrm{Li}>\mathrm{PBO}+\mathrm{Li}$ \\
\hline Berwaerts et al., 2011 & $\begin{array}{l}\text { PER + SH (197) } \\
\text { PBO + SH (205) }\end{array}$ & $\mathrm{PER}+\mathrm{SH}=\mathrm{PBO}+\mathrm{SH}$ \\
\hline Szegedi et al., 2012 & $\begin{array}{l}\mathrm{ASE}+\mathrm{SH}(158) \\
\mathrm{PBO}+\mathrm{SH}(166)\end{array}$ & $\mathrm{ASE}+\mathrm{SH}>\mathrm{PBO}+\mathrm{SH}$ \\
\hline Ouyang et al., 2012 & $\begin{array}{l}\text { RSP + VPA (22) } \\
\text { HAL + VPA (19) }\end{array}$ & $\mathrm{RSP}+\mathrm{VPA}>\mathrm{HAL}+\mathrm{VPA}$ \\
\hline
\end{tabular}

$>=$ combinaison plus efficace que la monothérapie; $\mathrm{ALP}=$ allopurinol $; \mathrm{APT}=$ antipsychotique typique; $\mathrm{ARI}=$ aripiprazole; $\mathrm{ASE}=$ asenapine; $\mathrm{BZD}=$ benzodiazepines; $\mathrm{CBZ}=$ carbamazepine; $\mathrm{GBP}$ = gabapentine; $\mathrm{HAL}=$ haloperidol $; \mathrm{LAM}=$ lamotrigine; $\mathrm{LEV}=$ levomepromazine $; \mathrm{Li}=$ lithium; $\mathrm{NS}=$ non spécifié $;$ OLZ = olanzapine; $\mathrm{PBO}=$ placebo PER = palipéridone $; \mathrm{QTP}=$ quetiapine $; \mathrm{RSP}=$ risperidone; $\mathrm{SH}=$ stabilisateur de l'humeur (lithium or valproate); $\mathrm{TXF}=$ tamoxiphène $; \mathrm{VPA}=$ valproate. 
Ces études montrent également l'intérêt de ces associations sur du moyen terme (généralement de 1 à 2 ans) car elles augmentent le délai de récidive dépressive ou maniaque en comparaison avec un thymorégulateur en monothérapie malgré des profils de tolérance moins bons bien qu'acceptables [22].

Des questions se posent alors : doit-on poursuivre l'association ou favoriser une monothérapie comme traitement prophylactique? Si c'est le cas, quel est le traitement à enlever en première intention?

Les recommandations futures devront y répondre et proposer des algorithmes spécifiques pour ces combinaisons thérapeutiques. Dans l'attente, l'attitude thérapeutique sera à adapter au cas par cas, aux molécules utilisées et au profil clinique de la pathologie dont par exemple le type de polarité prédominante du patient [22] ou l'âge de début de la maladie [53]. Soulignons qu'au décours d'un premier épisode, les connaissances actuelles nous invitent à privilégier la monothérapie du fait de la moins bonne tolérance des associations.

\subsubsection{Traitement des états mixtes}

La prise en charge d'un état mixte est moins consensuelle et il existe globalement deux types de recommandations. Les premières qui recommandent l'utilisation préférentielle de certaines molécules considérées comme plus efficace dans cette indication spécifique. Ainsi elles préconisent le VPA, la carbamazepine et certains APA et considèrent le lithium comme moins efficace $[4,21,24]$.

Les secondes dont les recommandations professionnelles du NICE ont un positionnement différent en ne différenciant pas le traitement d'une manie classique de celui de l'état mixte [4]. Elles ne proposent pas de choix de molécules spécifiques hormis le fait d'éviter l'emploi d'un antidépresseur comme indiqué dans les autres recommandations.

Des études complémentaires spécifiques de ces états mixtes et bien menées sont nécessaires afin de proposer des recommandations avec un niveau de preuve acceptable.

\section{Conclusion}

Les troubles bipolaires sont des troubles évoluant sur la vie entière et dont le devenir, au delà des récurrences thymiques, est conditionné par la présence de symptômes résiduels et de troubles comorbides tant psychiatriques que somatiques. La prise en charge devra tenir compte de l'ensemble de ces éléments afin de promouvoir une bonne qualité de vie pour le patient. Ainsi, le traitement des épisodes aigus devra répondre aux impératifs d'efficacité tout 
en tenant compte de la nécessité de poursuivre le traitement à titre prophylactique et donc aussi d'un profil de tolérance acceptable au long cours pour le patient.

L'utilisation des recommandations a démontré que l'utilisation systématique par les cliniciens d'algorithmes décisionnels en comparaison à une modalité de traitement habituel (« treatment as usual ») améliore la prise en charge globale des patients bipolaires [54]. Pourtant ces recommandations ont leurs propres limites et elles ne répondent pas à toutes les questions des cliniciens qui sont confrontés à des présentations cliniques et des réponses thérapeutiques très hétérogènes. Il conviendrait de compléter les données issues des essais cliniques par des données issues de cohortes pour aller vers une médecine personnalisée tenant compte plus spécifiquement des profils évolutifs de la maladie et des comorbidités. Bien évidemment aucune recommandation ne saurait répondre à la singularité de chaque situation clinique et ne saurait faire oublier l'expérience clinique dans la prise de décision.

\section{Bibliographie :}

1. Murray C, Lopez A. The Global Burden of Disease. A Comprehensive Assessment of Morbidity and Disability From Diseases, Injuries and Risk Factors in 1990 and Projected to 2020. Cambridge, Massachusetts: Harvard University Press; 1996.

2. Geoffroy PA, Etain B, Scott J, et al. Reconsideration of bipolar disorder as a developmental disorder: Importance of the time of onset. J Physiol Paris. 2013; 107(4):278-85.

3. Scott J, Leboyer M. Consequences of delayed diagnosis of bipolar disorders. Encephale. 2011;37 Suppl 3:S173- 175.

4. NICE. Bipolar disorder [Internet]. NICE. 2006 [cité 28 janv 2012]. Disponible sur: http://www.nice.org.uk/

5. Yatham LN, Kennedy SH, Schaffer A, et al. Canadian Network for Mood and Anxiety Treatments (CANMAT) and International Society for Bipolar Disorders (ISBD) collaborative update of CANMAT guidelines for the management of patients with bipolar disorder: update 2009. Bipolar Disord. 2009;11(3):225- 255.

6. Grunze H, Vieta E, Goodwin GM, et al. The World Federation of Societies of Biological Psychiatry (WFSBP) guidelines for the biological treatment of bipolar disorders: update 2009 on the treatment of acute mania. World J Biol Psychiatry. 2009;10(2):85- 116.

7. Goodwin GM. Evidence-based guidelines for treating bipolar disorder: revised second edition--recommendations from the British Association for Psychopharmacology. $\mathbf{J}$ Psychopharmacol. (Oxford). 2009;23(4):346- 388.

8. Malhi GS, Adams D, Lampe L, et al. Clinical practice recommendations for bipolar disorder. Acta Psychiatr Scand Suppl. 2009;(439):27- 46.

9. American Psychiatric Association. Practice guideline for the treatment of patients with bipolar disorder (revision). Am J Psychiatry. 2002;159(4 Suppl):1- 50.

10. Grunze H, Kasper S, Goodwin G, et al. The World Federation of Societies of Biological Psychiatry (WFSBP) Guidelines for the Biological Treatment of Bipolar Disorders, Part II: Treatment of Mania. World J Biol Psychiatry. 2003;4(1):5- 13. 
11. Grunze H, Kasper S, Goodwin G, et al. The World Federation of Societies of Biological Psychiatry (WFSBP) guidelines for the biological treatment of bipolar disorders, part III: maintenance treatment. World J Biol Psychiatry. 2004;5(3):120- 135.

12. Goodwin GM. Evidence-based guidelines for treating bipolar disorder: recommendations from the British Association for Psychopharmacology. J Psychopharmacol. 2003;17(2):149- 173.

13. Llorca P-M, Courtet P, Martin P, et al. [Screening and management of bipolar disorders: results]. Encephale. 2010;36 Suppl 4:S86- 102.

14. Yatham LN, Kennedy SH, O'Donovan C, et al. Canadian Network for Mood and Anxiety Treatments (CANMAT) guidelines for the management of patients with bipolar disorder: consensus and controversies. Bipolar Disord. 2005;7 Suppl 3:5- 69.

15. HAS. Troubles Bipolaires. Guide Médecin - AL23. 1-74, 2009.

16. Johnson FR, Ozdemir S, Manjunath R, et al. Factors that affect adherence to bipolar disorder treatments: a stated-preference approach. Med Care. 2007;45(6):545- 552.

17. Perlis RH, Ostacher MJ, Patel JK, et al. Predictors of recurrence in bipolar disorder: primary outcomes from the Systematic Treatment Enhancement Program for Bipolar Disorder (STEP-BD). Am J Psychiatry. 2006;163(2):217- 224.

18. Schneck CD, Miklowitz DJ, Miyahara S, et al. The prospective course of rapid-cycling bipolar disorder: findings from the STEP-BD. Am J Psychiatry. 2008;165(3):370- 377.

19. McKnight RF, Adida M, Budge $\mathrm{K}$, et al. Lithium toxicity profile: a systematic review and meta-analysis. Lancet. 2012;379(9817):721- 728.

20. Tomson T, Battino D. Teratogenic effects of antiepileptic drugs. Lancet Neurol. 2012;11(9):803- 813.

21. Tohen M, Goldberg JF, Gonzalez-Pinto Arrillaga AM, et al. A 12-week, double-blind comparison of olanzapine vs haloperidol in the treatment of acute mania. Arch Gen Psychiatry. 2003;60(12):1218- 1226.

22. Geoffroy PA, Etain B, Henry C, et al. Combination Therapy for Manic Phases: A Critical Review of a Common Practice. CNS Neurosci Ther. 2012;18(12):957-64.

23. Nivoli AMA, Murru A, Goikolea JM, et al. New treatment guidelines for acute bipolar mania: A critical review. J Affect Disord. 2012;140(2):125-41.

24. Lim PZ, Tunis SL, Edell WS, et al. Medication prescribing patterns for patients with bipolar I disorder in hospital settings: adherence to published practice guidelines. Bipolar Disord. 2001;3(4):165- 173.

25. Wolfsperger M, Greil W, Rössler W, et al. Pharmacological treatment of acute mania in psychiatric in-patients between 1994 and 2004. J Affect Disord. 2007;99(1-3):9- 17.

26. Haro JM, Reed C, Gonzalez-Pinto A, et al. 2-Year course of bipolar disorder type I patients in outpatient care: factors associated with remission and functional recovery. Eur Neuropsychopharmacol. 2011;21(4):287- 293.

27. Cookson J. Use of antipsychotic drugs and lithium in mania. $\mathrm{Br} \mathrm{J}$ Psychiatry. 2001;178(Suppl 41):S148- 156.

28. Smith LA, Cornelius V, Warnock A, et al. Pharmacological interventions for acute bipolar mania: a systematic review of randomized placebo-controlled trials. Bipolar Disord. 2007;9(6):551- 560.

29. Garfinkel PE, Stancer HC, Persad E. A comparison of haloperidol, lithium carbonate and their combination in the treatment of mania. J Affect Disord. déc 1980;2(4):279- 288.

30. Klein E, Bental E, Lerer B, et al. Carbamazepine and haloperidol $\mathrm{v}$ placebo and haloperidol in excited psychoses. A controlled study. Arch Gen Psychiatry. 1984;41(2):165- 170.

31. Muller AA, Stoll KD. Carbamazepine and oxcarbazepine in the treatment of manic syndromes: studies in Germany. In: Emrich HM, Okuma T, Muller AA, eds. 
Anticonvulsants in Affective Disorders. Amsterdam, Netherlands: Excerpta Medica; 1984:134-147.

32. Desai NG, Gangadhar BN, Channabasavanna SM. et al. Carbamazepine hastens therapeutic action of lithium in mania. Proceedings Inter-national Conference on New Directions in Affective Disorders. Jerusalem, Israel, 1987:97. Abstract.

33. Möller HJ, Kissling W, Riehl T, Bäuml J, Binz U, Wendt G. Doubleblind evaluation of the antimanic properties of carbamazepine as a comedication to haloperidol. Prog. Neuropsychopharmacol. Biol Psychiatry. 1989;13(1-2):127- 136.

34. Anand, A., Oren, DA, Berman, RM, et al (1999). Lamotrigine treatment of lithium failure outpatient mania: a double-blind, placebo-controlled trial. In JC Scares \& S. Gershon (Eds.), Abstract Book on 3rd international conference on Bipolar disorder (p.23). Pittsburgh, PA. Copenhagen: Munksgaard.

35. Müller-Oerlinghausen B, Retzow A, et al. Valproate as an adjunct to neuroleptic medication for the treatment of acute episodes of mania: a prospective, randomized, double-blind, placebo-controlled, multicenter study. European Valproate Mania Study Group. J Clin Psychopharmacol. 2000;20(2):195- 203.

36. Pande AC, Crockatt JG, Janney CA, et al. Gabapentin in bipolar disorder: a placebocontrolled trial of adjunctive therapy. Gabapentin Bipolar Disorder Study Group. Bipolar Disord. 2000;2(3 Pt 2):249- 255.

37. Sachs GS, Grossman F, Ghaemi SN, et al. Combination of a mood stabilizer with risperidone or haloperidol for treatment of acute mania: a double-blind, placebocontrolled comparison of efficacy and safety. Am J Psychiatry. 2002;159(7):1146- 1154.

38. Tohen M, Chengappa KNR, Suppes T, et al. Efficacy of olanzapine in combination with valproate or lithium in the treatment of mania in patients partially nonresponsive to valproate or lithium monotherapy. Arch Gen Psychiatry. 2002;59(1):62- 69.

39. Delbello MP, Schwiers ML, Rosenberg HL, et al. A double-blind, randomized, placebocontrolled study of quetiapine as adjunctive treatment for adolescent mania. J Am Acad Child Adolesc Psychiatry. 2002;41(10):1216- 1223.

40. Yatham LN, Grossman F, Augustyns I, et al. Mood stabilisers plus risperidone or placebo in the treatment of acute mania. International, double-blind, randomised controlled trial. Br J Psychiatry. 2003;182:141- 147.

41. Sachs G, Chengappa KNR, Suppes T, et al. Quetiapine with lithium or divalproex for the treatment of bipolar mania: a randomized, double-blind, placebo-controlled study. Bipolar Disord. 2004;6(3):213- 223.

42. McIntyre RS, Konarski JZ, Jones M, et al. Quetiapine in the treatment of acute bipolar mania: efficacy across a broad range of symptoms. J Affect Disord. 2007;100 Suppl 1:S5- 14.

43. Yatham LN, Vieta E, Young AH, et al. A double blind, randomized, placebo-controlled trial of quetiapine as an add-on therapy to lithium or divalproex for the treatment of bipolar mania. Int Clin Psychopharmacol. 2007;22(4):212- 220.

44. Vieta E, T'joen C, McQuade RD, et al. Efficacy of adjunctive aripiprazole to either valproate or lithium in bipolar mania patients partially nonresponsive to valproate/lithium monotherapy: a placebo-controlled study. Am J Psychiatry. 2008;165(10):1316- 1325 .

45. Juruena MF, Ottoni GL, Machado-Vieira R, et al. Bipolar I and II disorder residual symptoms: oxcarbazepine and carbamazepine as add-on treatment to lithium in a doubleblind, randomized trial. Prog. Neuropsychopharmacol. Biol. Psychiatry. 2009;33(1):94- 99. 
46. Szegedi A, Calabrese JR, Stet L, et al. Asenapine as Adjunctive Treatment for Acute Mania Associated With Bipolar Disorder: Results of a 12-Week Core Study and 40Week Extension. Journal of Clinical Psychopharmacology. 2012;32(1):46- 55.

47. Akhondzadeh S, Milajerdi MR, Amini H, et al. Allopurinol as an adjunct to lithium and haloperidol for treatment of patients with acute mania: a double-blind, randomized, placebo-controlled trial. Bipolar Disord. 2006;8(5 Pt 1):485- 489.

48. Sussman N, Mullen J, Paulsson B, et al. Rates of remission/euthymia with quetiapine in combination with lithium/divalproex for the treatment of acute mania. J Affect Disord. 2007;100 Suppl 1:S55- 63.

49. Tohen M, Bowden CL, Smulevich AB, et al. Olanzapine plus carbamazepine v. carbamazepine alone in treating manic episodes. Br J Psychiatry. 2008;192(2):135- 143.

50. Amrollahi Z, Rezaei F, Salehi B, et al. Double-blind, randomized, placebo-controlled 6week study on the efficacy and safety of the tamoxifen adjunctive to lithium in acute bipolar mania. J Affect Disord. 2011;129(1-3):327- 331.

51. Berwaerts J, Lane R, Nuamah IF, et al. Paliperidone extended-release as adjunctive therapy to lithium or valproate in the treatment of acute mania: a randomized, placebocontrolled study. J Affect Disord. 2011;129(1-3):252- 260.

52. Ouyang W-C, Hsu M-C, Yeh I-N, et al. Efficacy and safety of combination of risperidone and haloperidol with divalproate in patients with acute mania. Int $\mathbf{J}$ Psychiatry Clin Pract. 2012;16(3):178-88.

53. Geoffroy PA, Etain B, Leboyer $\mathrm{M}$, et al. Une entité clinique aux implications thérapeutiques majeures: le trouble bipolaire à début précoce. Annales Médicopsychologiques. 2012;170(7):502- 509 .

54. Bauer MS, Biswas K, Kilbourne AM. Enhancing multiyear guideline concordance for bipolar disorder through collaborative care. Am J Psychiatry. 2009;166(11):1244- 1250. 